\title{
Rare Presentation of Deep Vein Thrombosis and Submassive Pulmonary Emboli Due to Hypercoagulable State With Supratherapeutic Anticoagulation
}

\author{
Mark D. Rivera-Morales 1, 2 , Jesse C. Wu ${ }^{1,2}$, Larissa Dub 3, 2, 1, Latha Ganti 4, 3, 2, 5 \\ 1. Emergency Medicine, University of Central Florida Hospital Corporation of America (HCA) Healthcare Graduate \\ Medical Education Consortium Emergency Medicine Residency Program of Greater Orlando, Orlando, USA 2. \\ Emergency Medicine, Osceola Regional Medical Center, Kissimmee, USA 3. Emergency Medicine, University of Central \\ Florida College of Medicine, Orlando, USA 4. Emergency Medicine, Envision Physician Services, Plantation, USA 5. \\ Emergency Medicine, University of Central Florida Hospital Corporation of America (HCA) Healthcare Graduate \\ Medical Education Consortium Emergency Medicine Residency Program of Greater Orlando, Olrando, USA
}

Corresponding author: Latha Ganti, latha.ganti@ucf.edu

\begin{abstract}
We present a case of an elderly male with multiple co-morbidities, including atrial fibrillation on warfarin and recently diagnosed left lower extremity deep vein thrombosis (DVT), who presented to the emergency department for dyspnea. He was found to be hypoxic and mildly hypotensive. He was diagnosed with submassive pulmonary emboli (PE) despite having a supratherapeutic international normalized ratio (INR). In this case report, the clinical presentation, diagnostic workup, and management of this patient are discussed.
\end{abstract}

Categories: Emergency Medicine, Hematology

Keywords: supratherapeutic inr, thromboembolism, deep venous thrombosis, pulmonary embolism, hypercoagulable

\section{Introduction}

Approximately 200,000 people are diagnosed with pulmonary emboli (PE) each year in the United States, and most are derived from a lower extremity deep vein thrombosis (DVT). About $4-5 \%$ of the cases of PE will develop shock and $45 \%$ of those cases with shock will die [1]. Virchow's triad (stasis, vascular injury, and hypercoagulability) is classically taught as the origin of thromboembolism, including PE. Common risk factors for PE include recent major surgery, trauma, prolonged immobility, malignancy, chronic inflammatory or autoimmune disorder, infection, congenital hypercoagulable state (e.g., factor V Leiden mutation), old age, pregnancy, and oral contraceptive use. Patients with PE commonly present with dyspnea on exertion, pleuritic chest pain, cough, orthopnea, hemoptysis, and leg pain or swelling. Upon examination, these patients may have tachypnea, tachycardia, and unilateral calf swelling or tenderness.

Review began 07/16/2021 Review ended 08/08/2021 Published 08/19/2021

\section{○ Copyright 2021}

Rivera-Morales et al. This is an open access article distributed under the terms of the Creative Commons Attribution License CC-BY 4.0., which permits unrestricted use, distribution, and reproduction in any medium, provided the original author and source are credited.
Electrocardiogram (ECG) and chest radiograph (CXR) findings are often nonspecific, with sinus tachycardia being the most common abnormal ECG finding and Westermark sign and Hampton's hump as the classic CXR findings in PE.

Several decision tools are available to risk stratify patients suspected to have PE, such as the Well's criteria and Revised Geneva Score. In combination with the d-dimer assay, these tools are useful in ruling out patients with low to moderate risk for PE. Computed tomography pulmonary angiography (CTPA) has been the gold standard in diagnosing PE with ventilation-perfusion (V/Q) scan reserved for those patients who have impaired renal function or are allergic to contrast. In addition, bedside ultrasound has also been a useful adjunct in diagnosing PE, especially at identifying those patients with significant right ventricular (RV) dysfunction. Patients with low-risk PE determined by the PE Severity Index (PESI) can be safely discharged with direct oral anticoagulation without an increase in mortality, recurrent PE, or major bleeding [2]. For stable patients who require admission, low molecular weight heparin is preferred due to its low risk of bleeding, death, and lower cost [3].

Submassive PE is defined as patients with systolic blood pressure $>90 \mathrm{mmHg}$ but with signs of RV dysfunction either on ultrasonography or as evidenced by elevated troponin or B-natriuretic peptide (BNP). The cornerstone of treatment is to initiate anticoagulation and hemodynamic support with supplemental oxygenation to maintain normoxemia, judicious fluid support, vasopressor to support blood pressure, and pulmonary vasodilator to improve pulmonary vascular resistance [4]. In massive PE (patients with hemodynamic decompensation), thrombolysis has been deemed an effective treatment if there are no contraindications $[5,6]$. The benefits of thrombolysis in submassive are less clear because there is still a risk of intracranial hemorrhage (ICH) [7,8]. Catheter-directed thrombolysis may be an expensive option for patients who are 65 years or older with increased risk of ICH or major bleeding at the expense of increased intensive care unit (ICU) length of stay (LOS) $[9,10]$. In patients with massive PE, surgical embolectomy may 


\section{Cureus}

be an option if the patient has a contraindication to thrombolysis or is unresponsive to thrombolysis [11]. Finally, extracorporeal membrane oxygenation (ECMO) may be the last resort for patients with massive PE who have refractory shock, cardiac arrest, or contraindication for thrombolysis or surgical embolectomy [12].

\section{Case Presentation}

An 86-year-old male presented to our emergency department (ED) with complaints of shortness of breath (SOB) and dyspnea on minimal exertion. The patient had a past medical history significant for chronic obstructive pulmonary disease (COPD), congestive heart failure with preserved ejection fraction, and atrial fibrillation on anticoagulation with warfarin, hypertension, dyslipidemia, a cerebrovascular accident without any residual deficits, seizures, benign prostatic hyperplasia (BPH), and a recently diagnosed left lower extremity DVT. The patient presented via EMS to our emergency department from home as a "CPAP alert." The patient was placed on CPAP en route to the hospital because, at home, he had a $\mathrm{SpO}_{2}$ of $77 \%$ on room air and was notably short of breath. The patient's respiratory status rapidly improved with CPAP and upon arrival to the ED, he was placed on supplemental oxygen via nasal cannula at 4 liters per minute and was saturating well with $\mathrm{SpO}_{2}$ of $98 \%$. Upon further history, the patient endorsed dyspnea, in addition to left leg pain and swelling for the past month. He did not seek earlier medical care secondary to other issues at home. He also reported intermittent abdominal pain associated with eating as well as watery diarrhea for the past month. He denied fever, chest pain, nausea, vomiting, syncope, or any other complaints on review of systems.

In the ED, the patient was found to have a left lower extremity DVT, bilateral pulmonary embolism diagnosed by CTPA, as well as a left atrial appendage thrombus (Figures 1-2).

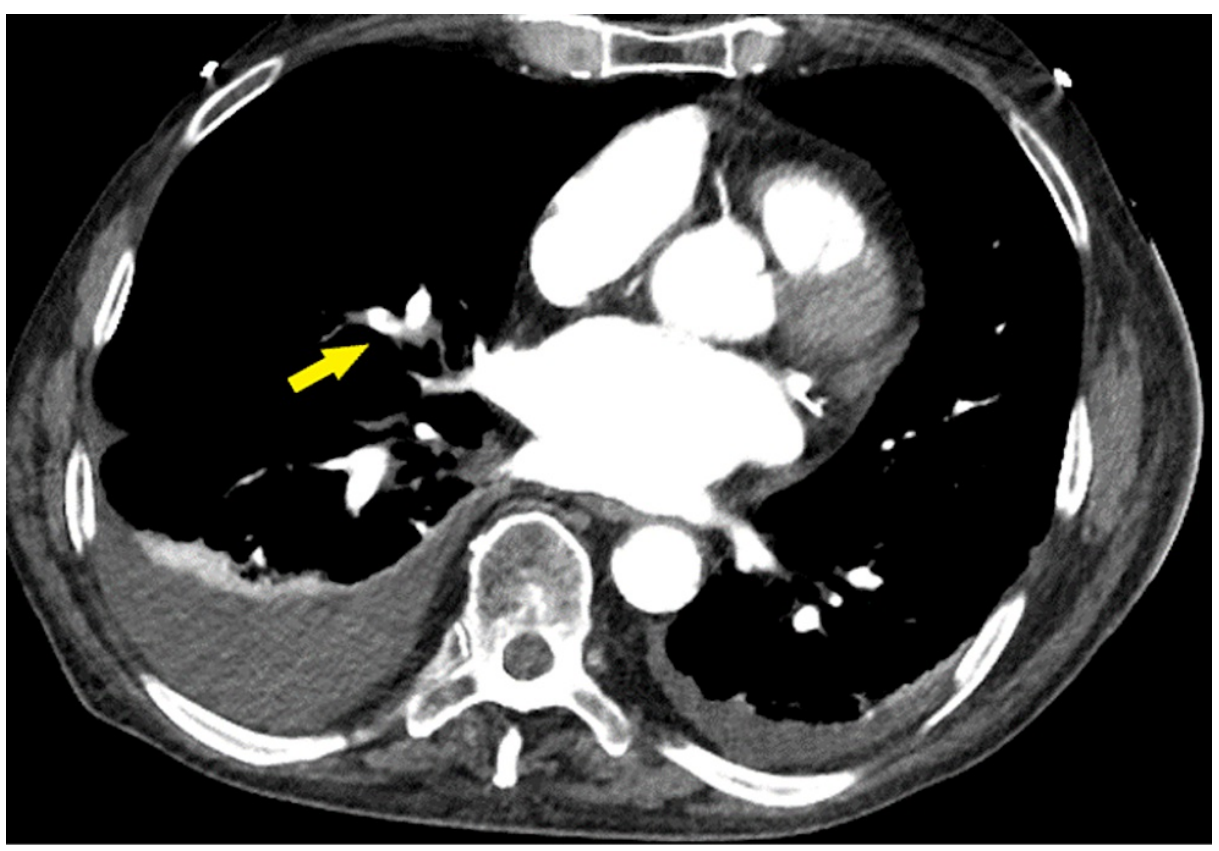

FIGURE 1: Computed tomography angiography showing pulmonary embolism, identified in the right middle lobe pulmonary arterial segments (yellow arrow). 


\section{Cureus}

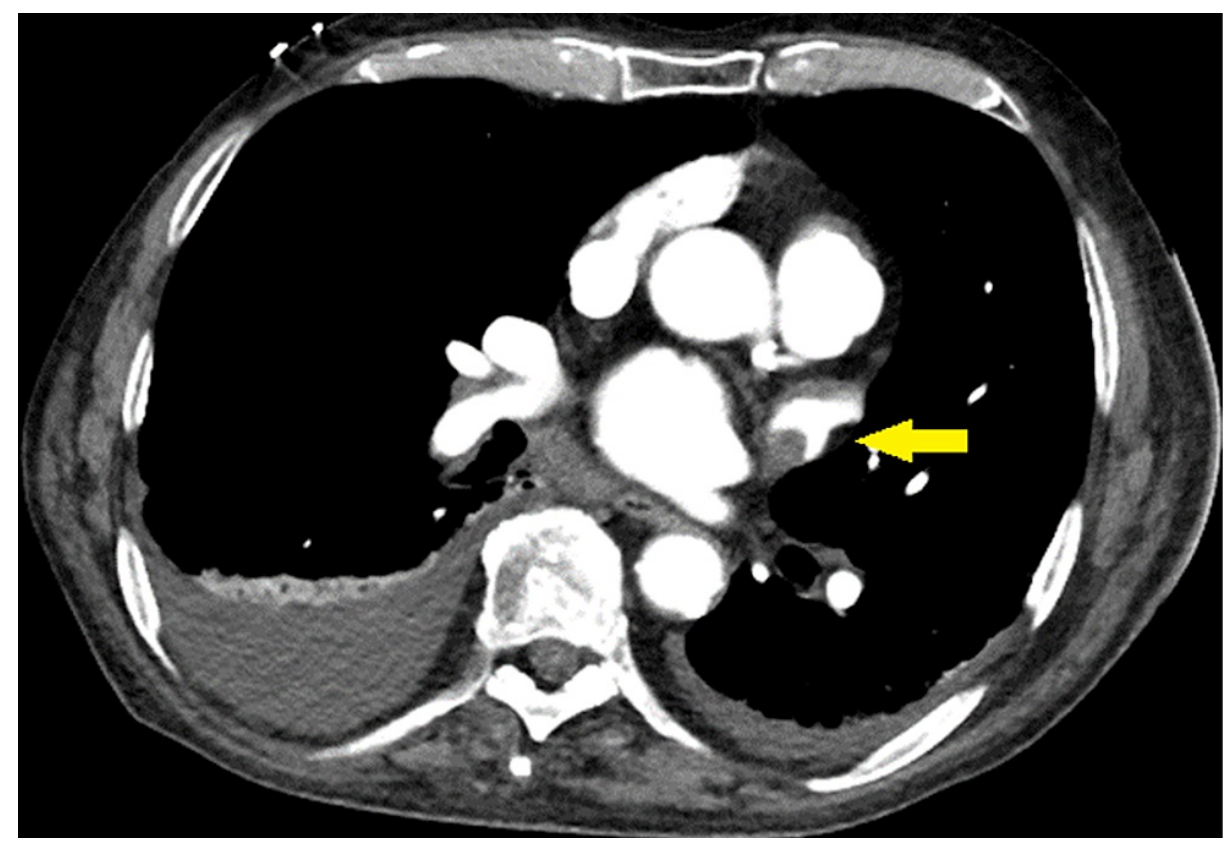

FIGURE 2: Computed tomography pulmonary angiography showing a left atrial appendage thrombus (yellow arrow).

Labs were significant for a supratherapeutic INR of 7.5 and an elevated activated partial thromboplastin time (aPTT) of 54.7 seconds. Also, N-terminal proBNP levels were elevated at $2831 \mathrm{pg} / \mathrm{ml}$, troponin-I was not elevated. Twelve-lead ECG showed atrial fibrillation with a normal rate. A cardiologist was consulted, who recommended initiation of subcutaneous enoxaparin despite a supratherapeutic INR and vitamin K reversal with oral phytonadione. The plan included transitioning the patient to a direct oral anticoagulant (DOAC), such as apixaban, prior to discharge. Hematology was consulted to weigh in on the evaluation of this hypercoagulable state despite being appropriately anticoagulated with warfarin. The patient was admitted to the cardiovascular ICU in stable but guarded condition.

\section{Discussion}

PE is a potentially life-threatening diagnosis that cannot be missed in the ED, especially when a patient with risk factors presents with signs and symptoms that suggest PE. It is well known that PE can lead to hypoxemia via V/Q mismatch, which can increase pulmonary vascular resistance and subsequently further impair RV function and cardiac output [13]. Therefore, it is important to provide supplemental oxygenation, such as a nasal cannula or non-invasive ventilation, in patients who are hypoxic. However, PE patients, especially those with submassive and massive PE, are extremely preload dependent. Thus, it is paramount to try to avoid any interventions that could increase intrathoracic pressure that further depresses the preload, such as endotracheal intubation. In patients who are already hypotensive or have hemodynamic decompensation, intubation can lead to cardiac arrest [14]. Fortunately, this patient only required nasal cannula oxygenation to maintain his oxygen saturation. It is remarkable that this patient was well appearing without obvious respiratory distress despite his initial hypoxemia that required non-invasive ventilation. Clinicians must always remain vigilant because patients with submassive PE can decompensate at any time.

Intravenous (IV) fluid administration is the traditional teaching to improve preload. However, fluid loading must be judicious in the CHF patient to prevent fluid overloading. In addition, there are some studies that suggest IV fluid administration may actually worsen RV function and impair cardiac output in patients who already have severe RV dysfunction [15]. In this case, volume off-loading with diuretics has been advocated to improve RV function in patients with submassive PE [16]. In patients with acute hemodynamic decompensation, vasopressors such as norepinephrine should be administered to improve cardiac output and coronary perfusion, especially if the patients have contraindications to thrombolysis [4,17]. More recently, pulmonary vasodilators, such as inhaled nitric oxide, have been demonstrated to decrease pulmonary vascular resistance, $\mathrm{RV}$ afterload, and improve V/Q mismatch [18]. It is a potential intervention in patients with shock refractory to vasopressor or thrombolysis. Even though our patient was borderline hypotensive, he was able to maintain his SBP >90 $\mathrm{mmHg}$ and has no signs of RV strain on bedside cardiac ultrasound. Therefore, vasopressor and thrombolysis were not necessary. Even if this patient were acutely decompensating, thrombolysis would have been contraindicated since he was on warfarin.

It is puzzling that this patient developed a DVT and bilateral PE despite being on warfarin with a supratherapeutic INR. However, cases of PE while on therapeutic or supratherapeutic anticoagulation have 


\section{Cureus}

been described in the literature [19]. Perhaps this patient had an undiagnosed underlying malignancy, as evidenced by the pulmonary nodules and enlarged retroperitoneal lymph nodes on CT, which contributed to his hypercoagulable state. There are multiple studies demonstrating that low molecular weight heparin and direct factor Xa inhibitors are superior to warfarin in terms of preventing recurrent venous

thromboembolism and decreasing the incidence of major bleeding [20]. Therefore, it makes sense to discontinue warfarin on this patient and continue this patient on low molecular weight heparin or apixaban upon discharge.

It is suspected that our patient likely has an undiagnosed malignancy as evidenced by the two pulmonary nodules, enlarged retroperitoneal lymph nodes, and small volume ascites found incidentally on CT imaging. This was believed to have contributed to his hypercoagulable state. The 2D echocardiogram done after admission did show evidence of mild RV dilation suggestive of RV dysfunction (Figure 3).

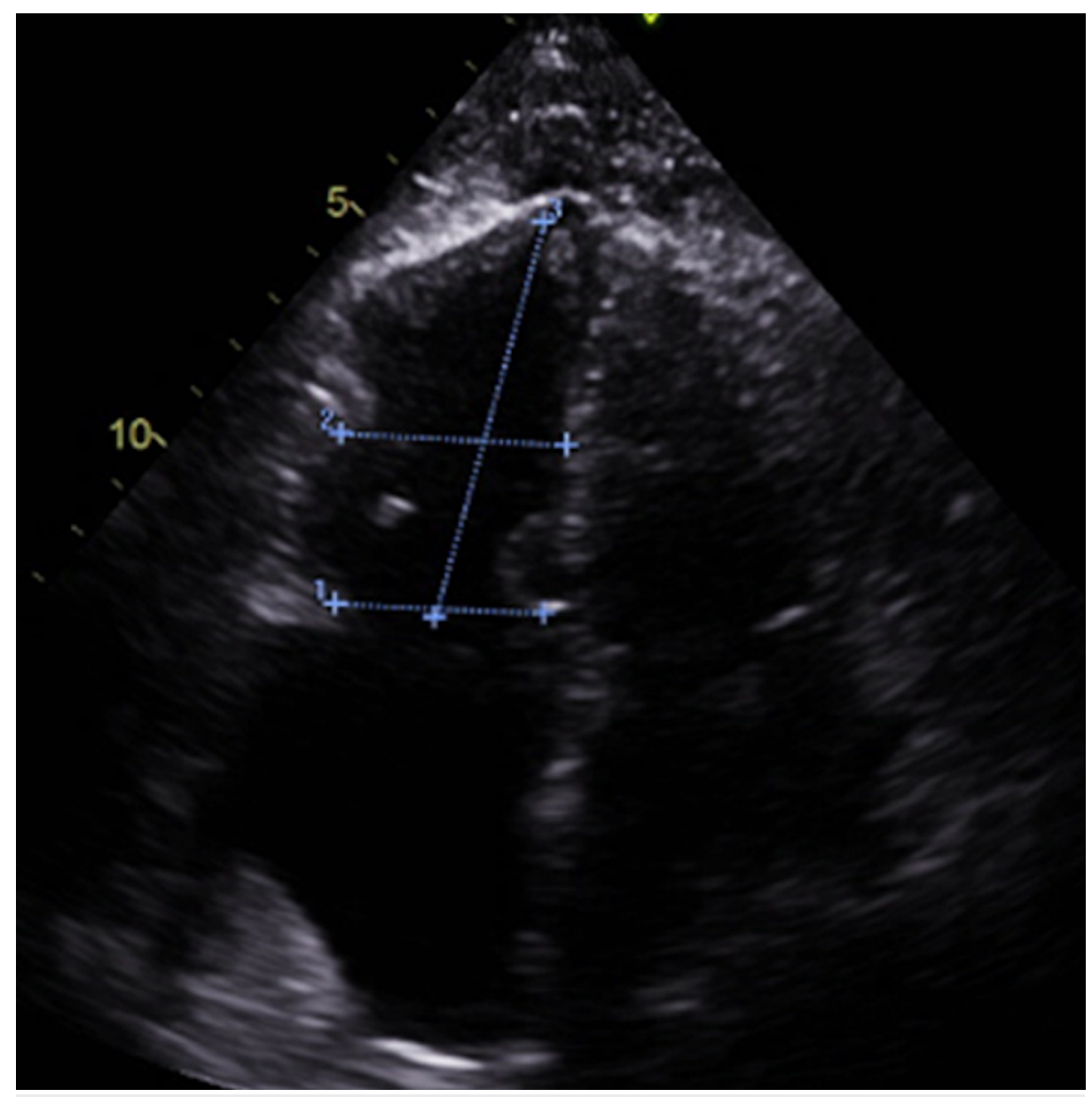

FIGURE 3: Two-dimensional echocardiogram showing RV dilation.

$\mathrm{A} 4 \mathrm{CH} \mathrm{RV}$ diameter was $3.7 \mathrm{~cm}$ suggestive of RV dysfunction. In comparison, the A4CH LV diameter was 3.1 $\mathrm{cm}$. RV: right ventricle, $\mathrm{A} 4 \mathrm{CH}$ : apical 4 chambers, LV: left ventricle.

The patient remained hemodynamically stable during his stay in the hospital. He subsequently was converted to oral apixaban and warfarin was discontinued. After discussion with the patient and family, the patient was discharged home with hospice care and outpatient follow-up with hematology was recommended.

\section{Conclusions}

Pulmonary embolism is a life-threatening diagnosis that should be considered in any patient who presents to the emergency department with symptoms of dyspnea, chest pain, palpitations, syncope, or unilateral leg swelling. Risk factors should be immediately reviewed and all presenting symptoms should be evaluated. Clinicians must balance the patient's delicate respiratory state while closely monitoring the patient's oxygen demands and respiratory drive. Fluid resuscitation must be judicious and close hemodynamic monitoring must be utilized in patients with massive and submassive PE. Early vasopressor support or thrombolysis should be considered in patients with no contraindications who present with refractory hypotension and hemodynamic instability. Finally, involving the appropriate subspecialists in 
patients who continue to experience thrombotic events despite therapeutic or supratherapeutic anticoagulation should be considered.

\section{Additional Information \\ Disclosures}

Human subjects: Consent was obtained or waived by all participants in this study. HCA Centralized Algorithms for Research Rules on IRB Exemptions (CARRIE)/IRB manager issued approval 2021-353. HCA Centralized Algorithms for Research Rules on IRB Exemptions (CARRIE)/IRB manager issued approval [2021-353]. Based on the information provided and attested as true, the research plan described does not require IRB oversight. This is because you are either (a) not engaging in research with human subjects as defined by federal regulations; (b) engaging in research with human subjects deemed excluded from IRB oversight per 45CFR46.102(l) OR (c) engaging in research with sufficient human subject protections in the design to meet one or more IRB exemption criteria set forth in 45CFR46.104. Conflicts of interest: In compliance with the ICMJE uniform disclosure form, all authors declare the following: Payment/services info: All authors have declared that no financial support was received from any organization for the submitted work. Financial relationships: All authors have declared that they have no financial relationships at present or within the previous three years with any organizations that might have an interest in the submitted work. Other relationships: All authors have declared that there are no other relationships or activities that could appear to have influenced the submitted work.

\section{Acknowledgements}

This research was supported (in whole or in part) by HCA Healthcare and/or an HCA Healthcare affiliated entity. The views expressed in this publication represent those of the author(s) and do not necessarily represent the official views of HCA Healthcare or any of its affiliated entities.

\section{References}

1. Kline JA: Venous thromboembolism including pulmonary embolism. Tintinalli's Emergency Medicine: A Comprehensive Study Guide. Tintinalli JE, Ma OJ, Yealy DM, et al. (ed): McGraw Hill, New York; 2020. 9th ed.:

2. Logan JK, Pantle H, Huiras P, Bessman E, Bright L: Evidence-based diagnosis and thrombolytic treatment of cardiac arrest or periarrest due to suspected pulmonary embolism. Am J Emerg Med. 2014, 32:789-96. 10.1016/j.ajem.2014.04.032

3. Meyer G, Vieillard-Baron A, Planquette B: Recent advances in the management of pulmonary embolism: focus on the critically ill patients. Ann Intensive Care. 2016, 6:19. 10.1186/s13613-016-0122-z

4. Maughan BC, Frueh L, McDonagh MS, Casciere B, Kline JA: Outpatient treatment of low-risk pulmonary embolism in the era of direct oral anticoagulants: a systematic review. Acad Emerg Med. 2021, 28:226-39. 10.1111/acem.14108

5. Wan S, Quinlan DJ, Agnelli G, Eikelboom JW: Thrombolysis compared with heparin for the initial treatment of pulmonary embolism: a meta-analysis of the randomized controlled trials. Circulation. 2004, 110:744-9. 10.1161/01.CIR.0000137826.09715.9C

6. Kuo WT, Banerjee A, Kim PS, et al.: Pulmonary embolism response to fragmentation, embolectomy, and catheter thrombolysis (PERFECT): initial results from a prospective multicenter registry. Chest. 2015, 148:667-73. 10.1378/chest.15-0119

7. Pasrija C, Kronfli A, Rouse M, et al.: Outcomes after surgical pulmonary embolectomy for acute submassive and massive pulmonary embolism: A single-center experience. J Thorac Cardiovasc Surg. 2018, 155:10951106.e2. 10.1016/j.jtcvs.2017.10.139

8. Corsi F, Lebreton G, Bréchot N, et al.: Life-threatening massive pulmonary embolism rescued by venoarterial-extracorporeal membrane oxygenation. Crit Care. 2017, 21:76. 10.1186/s13054-017-1655-8

9. Fernandes CJ, Luppino Assad AP, Alves-Jr JL, Jardim C, de Souza R: Pulmonary embolism and gas exchange. Respiration. 2019, 98:253-62. 10.1159/000501342

10. Belenkie I, Dani R, Smith ER, Tyberg JV: Effects of volume loading during experimental acute pulmonary embolism. Circulation. 1989, 80:178-88. 10.1161/01.cir.80.1.178

11. Mercat A, Diehl JL, Meyer G, Teboul JL, Sors H: Hemodynamic effects of fluid loading in acute massive pulmonary embolism. Crit Care Med. 1999, 27:540-4. 10.1097/00003246-199903000-00032

12. Schouver ED, Chiche O, Bouvier P, Doyen D, Cerboni P, Moceri P, Ferrari E: Diuretics versus volume expansion in acute submassive pulmonary embolism. Arch Cardiovasc Dis. 2017, 110:616-25. 10.1016/j.acvd.2017.01.016

13. Kline JA, Hernandez J, Garrett JS, Jones AE: Pilot study of a protocol to administer inhaled nitric oxide to treat severe acute submassive pulmonary embolism. Emerg Med J. 2014, 31:459-62. 10.1136/emermed-2013202426

14. Summerfield DT, Desai H, Levitov A, Grooms DA, Marik PE: Inhaled nitric oxide as salvage therapy in massive pulmonary embolism: a case series. Respir Care. 2012, 57:444-8. 10.4187/respcare.01373

15. Hickey C, Schwartz A, Sattler S: Acute pulmonary embolism and ischemic foot in the setting of supratherapeutic INR. Am J Emerg Med. 2017, 35:666.e3-4. 10.1016/j.ajem.2016.11.009

16. Liu MY, Ballard DW, Huang J, Rauchwerger AS, Reed ME, Bouvet SC, Vinson DR: Acute pulmonary embolism in emergency department patients despite therapeutic anticoagulation. West J Emerg Med. 2018, 19:510-6. 10.5811/westjem.2018.1.35586

17. Meyer G, Marjanovic Z, Valcke J, et al.: Comparison of low-molecular-weight heparin and warfarin for the secondary prevention of venous thromboembolism in patients with cancer: a randomized controlled study. 


\section{Cureus}

Arch Intern Med. 2002, 162:1729-35. 10.1001/archinte.162.15.1729

18. Guo JD, Rajpura J, Hlavacek P, et al.: Comparative clinical and economic outcomes associated with warfarin versus apixaban in the treatment of patients with venous thromboembolism in a large U.S commercial claims database. J Manag Care Spec Pharm. 2020, 26:1017-26. 10.18553/jmcp.2020.19311

19. Alzghari SK, Seago SE, Garza JE, et al.: Retrospective comparison of low molecular weight heparin vs. warfarin vs. oral Xa inhibitors for the prevention of recurrent venous thromboembolism in oncology patients: The Re-CLOT study. J Oncol Pharm Pract. 2018, 24:494-500. 10.1177/1078155217718382

20. Almutairi AR, Zhou L, Gellad WF, Lee JK, Slack MK, Martin JR, Lo-Ciganic WH: Effectiveness and safety of non-vitamin K antagonist oral anticoagulants for atrial fibrillation and venous thromboembolism: a systematic review and meta-analyses. Clin Ther. 2017, 39:1456-1478.e36. 10.1016/j.clinthera.2017.05.358 